\title{
Positive Solutions for $(n-1,1)$-Type Singular Fractional Differential System with Coupled Integral Boundary Conditions
}

\author{
Ying Wang, ${ }^{1,2}$ Lishan Liu, ${ }^{1,3}$ Xinguang Zhang, ${ }^{3,4}$ and Yonghong $\mathrm{Wu}^{3}$ \\ ${ }^{1}$ School of Mathematical Sciences, Qufu Normal University, Qufu, Shandong 273165, China \\ ${ }^{2}$ School of Science, Linyi University, Linyi, Shandong 276000, China \\ ${ }^{3}$ Department of Mathematics and Statistics, Curtin University of Technology, Perth, WA 6845, Australia \\ ${ }^{4}$ Department of Mathematics and Informational Science, Yantai University, Yantai, Shandong 264005, China
}

Correspondence should be addressed to Lishan Liu; lls@mail.qfnu.edu.cn

Received 19 April 2014; Revised 27 August 2014; Accepted 15 September 2014; Published 3 November 2014

Academic Editor: Marco Donatelli

Copyright (C) 2014 Ying Wang et al. This is an open access article distributed under the Creative Commons Attribution License, which permits unrestricted use, distribution, and reproduction in any medium, provided the original work is properly cited.

We study the positive solutions of the $(n-1,1)$-type fractional differential system with coupled integral boundary conditions. The conditions for the existence of positive solutions to the system are established. In addition, we derive explicit formulae for the estimation of the positive solutions and obtain the unique positive solution when certain additional conditions hold. An example is then given to demonstrate the validity of our main results.

\section{Introduction}

This paper is motivated by the boundary value problem

$$
\begin{gathered}
D_{0^{+}}^{5 / 2} u(t)+\frac{(\sin t+\cos t) \sqrt{u}}{\sqrt[3]{t(1-t) v}}=0, \\
D_{0^{+}}^{5 / 2} v(t)+\frac{\sqrt[3]{v}}{e^{t} \sqrt{t(1-t) u}}=0, \quad 0<t<1, \\
u(0)=u^{\prime}(0)=0, \\
u(1)=\frac{1}{2} \int_{0}^{1} v(t) d t, \\
v(0)=v^{\prime}(0)=0, \\
v(1)=\int_{0}^{1} u(t) d t^{2},
\end{gathered}
$$

which arises in a variety of disciplinary areas such as mechanics, chemical physics, mathematical biology, flows, fluid electrical networks, and viscoelasticity (see [1-6] and the references cited therein). In problem (1), the nonlinearity $f(t, x, y)$ may be singular at $t=0,1$ and $y=0 ; g(t, x, y)$ may be singular at $t=0,1$ and $x=0$.

Research on fractional order integrodifferential operators dates back to the end of the 19th century, when Riemann and Liouville introduced the first definition of the fractional derivative. However, this field of study started to become attractive to engineers only in the late 1960 s, when fractional derivative description of some real systems was observed. It was found that fractional operators are nonlocal and are more suitable for constructing models possessing memory effect in a long time period, and hence fractional differential equations possess many advantages.

In this paper, we consider the existence of positive solutions for a nonlinear singular fractional differential system with coupled boundary conditions:

$$
\begin{gathered}
D_{0^{+}}^{\alpha_{1}} u(t)+f(t, u(t), v(t))=0, \\
D_{0^{+}}^{\alpha_{2}} v(t)+g(t, u(t), v(t))=0, \quad 0<t<1, \\
u(0)=u^{\prime}(0)=\cdots=u^{(n-2)}=0, \\
u(1)=\mu_{1} \int_{0}^{1} v(s) d A_{1}(s),
\end{gathered}
$$




$$
\begin{gathered}
v(0)=v^{\prime}(0)=\cdots=v^{(n-2)}=0, \\
v(1)=\mu_{2} \int_{0}^{1} u(s) d A_{2}(s),
\end{gathered}
$$

where $n-1<\alpha_{i} \leq n, n \geq 2$, and $D_{0^{+}}^{\alpha_{i}}$ is the standard RiemannLiouville derivative. $\mu_{i}>0, A_{i}$ is right continuous on $[0,1)$, left continuous at $t=1$, and nondecreasing on $[0,1], A_{i}(0)=$ 0 , and $\int_{0}^{1} x(s) d A_{i}(s)$ denotes the Riemann-Stieltjes integrals of $x$ with respect to $A_{i}(i=1,2) . f:(0,1) \times[0,+\infty) \times$ $(0,+\infty) \rightarrow[0,+\infty)$ and $g:(0,1) \times(0,+\infty) \times[0,+\infty) \rightarrow$ $[0,+\infty)$ are two continuous functions, and $f(t, x, y)$ may be singular at $t=0,1$ and $y=0$, while $g(t, x, y)$ may be singular at $t=0,1$ and $x=0$.

Coupled boundary value problem arises naturally in the research of Sturm-Liouville problems, reaction-diffusion equations, mathematical biology, and so on. In recent years, there has been a significant development in ordinary and partial differential equations involving fractional derivatives with coupled boundary conditions, as shown by [7-16] and the references therein. By using the nonlinear alternative of the Leray and Schauder theorem and the Krasnoselskii fixed point theorem in a cone, Bai and Fang in [17] obtained some results of existence of positive solutions by considering the singular coupled system of nonlinear fractional differential equations:

$$
\begin{gathered}
D^{s} u+f(t, v)=0, \\
D^{p} v+g(t, u)=0, \quad 0<t<1,
\end{gathered}
$$

where $0<s, p<1, D^{s}, D^{p}$ are two standard RiemannLiouville fractional derivative, and $f, g:(0,1] \times[0,+\infty) \rightarrow$ $[0,+\infty)$ are two given continuous functions and are singular at $t=0$.

Wang et al. [18] study the following system of nonlinear fractional differential equations:

$$
\begin{gathered}
D_{0^{+}}^{\alpha} u(t)+f(t, v(t))=0, \\
D_{0^{+}}^{\beta} v(t)+g(t, u(t))=0, \quad 0<t<1, \\
u(0)=v(0)=0, \\
u(1)=a u(\xi), \\
v(1)=b v(\xi),
\end{gathered}
$$

where $1<\alpha, \beta<2,0 \leq a, b<1,0<\xi<1, f, g:[0,1] \times$ $[0,+\infty) \rightarrow[0,+\infty)$ are continuous functions, and $D_{0^{+}}^{\alpha}$, $D_{0^{+}}^{\beta}$ are two standard Riemann-Liouville fractional derivatives. By using the Banach fixed point theorem and the nonlinear alternative of Leray-Schauder type, the existence and uniqueness of a positive solution are obtained.

In this paper, we consider the existence and uniqueness of positive solutions for the singular system (2). The work presented in this paper has the following new features. Firstly, until now, coupled integral boundary value problems for fractional differential system as system (2) have seldom been considered when $f(t, x, y)$ may be singular at $t=0,1$ and $y=0$, and $g(t, x, y)$ may be singular at $t=0,1$ and $x=$ 0 . Also $\int_{0}^{1} x(s) d A_{i}(s)$ denotes the Riemann-Stieltjes integral, and thus system (2) includes the multipoint problems and integral problems as special cases. Secondly, by using the wellknown fixed point theorem due to Guo-Krasnoselskii, we not only obtain the existence of positive solutions for system (2), but also obtain the uniqueness of system (2).

A vector $(u, v)$ is said to be a positive solution of system (2) if and only if $(u, v)$ satisfies (2) and $u(t)>0, v(t)>0$ for any $t \in(0,1]$.

\section{Preliminaries and Lemmas}

In what follows, we present some necessary definitions about fractional calculus theory.

Definition 1 (see $[2,19])$. Let $\alpha>0$ and let $u$ be piecewise continuous on $(0,+\infty)$ and integrable on any finite subinterval of $[0,+\infty)$. Then, for $t>0$, we call

$$
I_{0^{+}}^{\alpha} u(t)=\frac{1}{\Gamma(\alpha)} \int_{0}^{t}(t-s)^{\alpha-1} u(s) d s
$$

the Riemann-Liouville fractional integral of $u$ of order $\alpha$.

Definition 2 (see $[2,19]$ ). The Riemann-Liouville fractional derivative of order $\alpha>0, n-1 \leq \alpha<n, n \in \mathbb{N}$, is defined as

$$
D_{0^{+}}^{\alpha} u(t)=\frac{1}{\Gamma(n-\alpha)}\left(\frac{d}{d t}\right)^{n} \int_{0}^{t}(t-s)^{n-\alpha-1} u(s) d s
$$

where $\mathbb{N}$ denotes the natural number set and the function $u(t)$ is $n$ times continuously differentiable on $[0,+\infty)$.

Lemma 3 (see [2]). Let $\alpha>0$; then,

$$
\begin{aligned}
& I_{0^{+}}^{\alpha} D_{0^{+}}^{\alpha} u(t) \\
& \quad=u(t)+c_{1} t^{\alpha-1}+c_{2} t^{\alpha-2}+\cdots+c_{n} t^{\alpha-n}
\end{aligned}
$$

where $c_{1}, c_{2}, \ldots, c_{n} \in(-\infty,+\infty)$ and $n$ is the smallest integer greater than or equal to $\alpha$.

Lemma 4. Let $h_{i} \in C(0,1) \cap L^{1}(0,1)(i=1,2)$, and the following condition $\left(\mathbf{H}_{0}\right)$ holds:

$\left(\mathbf{H}_{0}\right)$

$$
\begin{gathered}
k_{1}=\int_{0}^{1} t^{\alpha_{2}-1} d A_{1}(t)>0, \\
k_{2}=\int_{0}^{1} t^{\alpha_{1}-1} d A_{2}(t)>0, \\
1-\mu_{1} \mu_{2} k_{1} k_{2}>0 .
\end{gathered}
$$


Then the system subjected to the coupled boundary conditions

$$
\begin{gathered}
D_{0^{+}}^{\alpha_{1}} u(t)+h_{1}(t)=0, \\
D_{0^{+}}^{\alpha_{2}} v(t)+h_{2}(t)=0, \\
0<t<1, \\
u(0)=u^{\prime}(0)=\cdots=u^{(n-2)}=0, \\
u(1)=\mu_{1} \int_{0}^{1} v(s) d A_{1}(s), \\
v(0)=v^{\prime}(0)=\cdots=v^{(n-2)}=0, \\
v(1)=\mu_{2} \int_{0}^{1} u(s) d A_{2}(s)
\end{gathered}
$$

has an integral representation

$$
\begin{aligned}
& u(t)=\int_{0}^{1} K_{1}(t, s) h_{1}(s) d s+\int_{0}^{1} H_{1}(t, s) h_{2}(s) d s \\
& v(t)=\int_{0}^{1} K_{2}(t, s) h_{2}(s) d s+\int_{0}^{1} H_{2}(t, s) h_{1}(s) d s
\end{aligned}
$$

where

$$
\begin{gathered}
K_{1}(t, s)=\frac{\mu_{1} \mu_{2} k_{1} t^{\alpha_{1}-1}}{1-\mu_{1} \mu_{2} k_{1} k_{2}} \int_{0}^{1} G_{1}(t, s) d A_{2}(t)+G_{1}(t, s), \\
H_{1}(t, s)=\frac{\mu_{1} t^{\alpha_{1}-1}}{1-\mu_{1} \mu_{2} k_{1} k_{2}} \int_{0}^{1} G_{2}(t, s) d A_{1}(t), \\
K_{2}(t, s)=\frac{\mu_{2} \mu_{1} k_{2} t^{\alpha_{2}-1}}{1-\mu_{1} \mu_{2} k_{1} k_{2}} \int_{0}^{1} G_{2}(t, s) d A_{1}(t)+G_{2}(t, s), \\
H_{2}(t, s)=\frac{\mu_{2} t^{\alpha_{2}-1}}{1-\mu_{1} \mu_{2} k_{1} k_{2}} \int_{0}^{1} G_{1}(t, s) d A_{2}(t),
\end{gathered}
$$$$
G_{i}(t, s)
$$$$
=\frac{1}{\Gamma\left(\alpha_{i}\right)}\left\{\begin{array}{c}
{[t(1-s)]^{\alpha_{i}-1}} \\
-(t-s)^{\alpha_{i}-1}, 0 \leq s \leq t \leq 1, \quad i=1,2 . \\
{[t(1-s)]^{\alpha_{i}-1}, 0 \leq t \leq s \leq 1,}
\end{array}\right.
$$

Proof. By Lemma 3, the system (9) is equivalent to the following integral equations system:

$$
\begin{aligned}
& u(t)=u(1) t^{\alpha_{1}-1}+\int_{0}^{1} G_{1}(t, s) h_{1}(s) d s, \\
& v(t)=v(1) t^{\alpha_{2}-1}+\int_{0}^{1} G_{2}(t, s) h_{2}(s) d s .
\end{aligned}
$$

Integrating (13) and (14) with respect to $d A_{2}(t)$ and $d A_{1}(t)$, respectively, we have

$$
\begin{aligned}
& \int_{0}^{1} u(t) d A_{2}(t) \\
& \quad=u(1) \int_{0}^{1} t^{\alpha_{1}-1} d A_{2}(t)+\iint_{0}^{1} G_{1}(t, s) h_{1}(s) d s d A_{2}(t) \\
& \int_{0}^{1} v(t) d A_{1}(t) \\
& \quad=v(1) \int_{0}^{1} t^{\alpha_{2}-1} d A_{1}(t)+\iint_{0}^{1} G_{2}(t, s) h_{2}(s) d s d A_{1}(t)
\end{aligned}
$$

which yield

$$
\begin{aligned}
& \frac{1}{\mu_{1}} u(1)-k_{1} v(1)=\iint_{0}^{1} G_{2}(t, s) h_{2}(s) d s d A_{1}(t), \\
& -k_{2} u(1)+\frac{1}{\mu_{2}} v(1)=\iint_{0}^{1} G_{1}(t, s) h_{1}(s) d s d A_{2}(t) .
\end{aligned}
$$

It follows from

$$
\left|\begin{array}{cc}
\frac{1}{\mu_{1}} & -k_{1} \\
-k_{2} & \frac{1}{\mu_{2}}
\end{array}\right|=\frac{1-\mu_{1} \mu_{2} k_{1} k_{2}}{\mu_{1} \mu_{2}} \neq 0
$$

that the system of (16) has a unique solution, which can be represented as

$$
\begin{gathered}
u(1)=\frac{\mu_{1}}{1-\mu_{1} \mu_{2} k_{1} k_{2}} \\
\cdot\left(\iint_{0}^{1} G_{2}(t, s) h_{2}(s) d s d A_{1}(t)\right. \\
\left.\quad+\mu_{2} k_{1} \iint_{0}^{1} G_{1}(t, s) h_{1}(s) d s d A_{2}(t)\right), \\
v(1)=\frac{\mu_{2}}{1-\mu_{1} \mu_{2} k_{1} k_{2}} \\
\quad\left(\iint_{0}^{1} G_{1}(t, s) h_{1}(s) d s d A_{2}(t)\right. \\
\left.\quad+\mu_{1} k_{2} \iint_{0}^{1} G_{2}(t, s) h_{2}(s) d s d A_{1}(t)\right) .
\end{gathered}
$$


Substituting (18) into (13) and (14), we have

$$
\begin{aligned}
& u(t)=\frac{\mu_{1} t^{\alpha_{1}-1}}{1-\mu_{1} \mu_{2} k_{1} k_{2}} \\
& \cdot\left(\iint_{0}^{1} G_{2}(t, s) h_{2}(s) d s d A_{1}(t)\right. \\
& \left.+\mu_{2} k_{1} \iint_{0}^{1} G_{1}(t, s) h_{1}(s) d s d A_{2}(t)\right) \\
& +\int_{0}^{1} G_{1}(t, s) h_{1}(s) d s \\
& =\int_{0}^{1} K_{1}(t, s) h_{1}(s) d s+\int_{0}^{1} H_{1}(t, s) h_{2}(s) d s, \\
& v(t)=\frac{\mu_{2} t^{\alpha_{2}-1}}{1-\mu_{1} \mu_{2} k_{1} k_{2}} \\
& \cdot\left(\iint_{0}^{1} G_{1}(t, s) h_{1}(s) d s d A_{2}(t)\right. \\
& \left.+\mu_{1} k_{2} \iint_{0}^{1} G_{2}(t, s) h_{2}(s) d s d A_{1}(t)\right) \\
& +\int_{0}^{1} G_{2}(t, s) h_{2}(s) d s \\
& =\int_{0}^{1} K_{2}(t, s) h_{2}(s) d s+\int_{0}^{1} H_{2}(t, s) h_{1}(s) d s .
\end{aligned}
$$

So (10) holds. This completes the proof of the lemma.

Lemma 5. For $t, s \in[0,1]$, the functions $K_{i}(t, s)$ and $H_{i}(t, s)$ $(i=1,2)$ defined by (11) possess the following properties:

$$
\begin{gathered}
K_{1}(t, s), H_{2}(t, s) \leq \rho s(1-s)^{\alpha_{1}-1}, \\
K_{2}(t, s), H_{1}(t, s) \leq \rho s(1-s)^{\alpha_{2}-1}, \\
K_{1}(t, s), H_{1}(t, s) \leq \rho t^{\alpha_{1}-1}, \\
K_{2}(t, s), H_{2}(t, s) \leq \rho t^{\alpha_{2}-1}, \\
K_{1}(t, s) \geq \varrho t^{\alpha_{1}-1} s(1-s)^{\alpha_{1}-1}, \\
H_{2}(t, s) \geq \varrho t^{\alpha_{2}-1} s(1-s)^{\alpha_{1}-1}, \\
K_{2}(t, s) \geq \varrho t^{\alpha_{2}-1} s(1-s)^{\alpha_{2}-1}, \\
H_{1}(t, s) \geq \rho t^{\alpha_{1}-1} s(1-s)^{\alpha_{2}-1}
\end{gathered}
$$

where

$$
\begin{aligned}
& \rho=\max \left\{\frac{1}{\Gamma\left(\alpha_{1}-1\right)}\left(\frac{\mu_{1} \mu_{2} k_{1}}{1-\mu_{1} \mu_{2} k_{1} k_{2}} \int_{0}^{1} d A_{2}(t)+1\right),\right. \\
& \frac{\mu_{1}}{\Gamma\left(\alpha_{2}-1\right)\left(1-\mu_{1} \mu_{2} k_{1} k_{2}\right)} \int_{0}^{1} A_{1}(t), \\
& \frac{1}{\Gamma\left(\alpha_{2}-1\right)}\left(\frac{\mu_{2} \mu_{1} k_{2}}{1-\mu_{1} \mu_{2} k_{1} k_{2}} \int_{0}^{1} d A_{1}(t)+1\right), \\
& \left.\frac{\mu_{2}}{\Gamma\left(\alpha_{1}-1\right)\left(1-\mu_{1} \mu_{2} k_{1} k_{2}\right)} \int_{0}^{1} d A_{2}(t)\right\}, \\
& \varrho=\min \left\{\frac{\mu_{1} \mu_{2} k_{1}}{\Gamma\left(\alpha_{1}\right)\left(1-\mu_{1} \mu_{2} k_{1} k_{2}\right)} \int_{0}^{1}(1-t) t^{\alpha_{1}-1} d A_{2}(t)\right. \text {, } \\
& \frac{\mu_{1}}{\Gamma\left(\alpha_{2}\right)\left(1-\mu_{1} \mu_{2} k_{1} k_{2}\right)} \int_{0}^{1}(1-t) t^{\alpha_{2}-1} d A_{1}(t), \\
& \frac{\mu_{2} \mu_{1} k_{2}}{\Gamma\left(\alpha_{2}\right)\left(1-\mu_{1} \mu_{2} k_{1} k_{2}\right)} \int_{0}^{1}(1-t) t^{\alpha_{2}-1} d A_{1}(t), \\
& \left.\frac{\mu_{2}}{\Gamma\left(\alpha_{1}\right)\left(1-\mu_{1} \mu_{2} k_{1} k_{2}\right)} \int_{0}^{1}(1-t) t^{\alpha_{1}-1} d A_{2}(t)\right\} \text {. }
\end{aligned}
$$

Proof. By [20], for any $t, s \in[0,1]$, we have

$$
\begin{aligned}
& \frac{(1-t) t^{\alpha_{i}-1} s(1-s)^{\alpha_{i}-1}}{\Gamma\left(\alpha_{i}\right)} \\
& \leq G_{i}(t, s) \leq \frac{s(1-s)^{\alpha_{i}-1}}{\Gamma\left(\alpha_{i}-1\right)}, \quad i=1,2 .
\end{aligned}
$$

It follows from (11) and (25) that

$$
\begin{aligned}
& K_{1}(t, s) \\
& =\frac{\mu_{1} \mu_{2} k_{1} t^{\alpha_{1}-1}}{1-\mu_{1} \mu_{2} k_{1} k_{2}} \int_{0}^{1} G_{1}(t, s) d A_{2}(t)+G_{1}(t, s) \\
& \leq \frac{\mu_{1} \mu_{2} k_{1} t^{\alpha_{1}-1}}{1-\mu_{1} \mu_{2} k_{1} k_{2}} \int_{0}^{1} \frac{s(1-s)^{\alpha_{1}-1}}{\Gamma\left(\alpha_{1}-1\right)} d A_{2}(t)+\frac{s(1-s)^{\alpha_{1}-1}}{\Gamma\left(\alpha_{1}-1\right)} \\
& \leq \frac{1}{\Gamma\left(\alpha_{1}-1\right)}\left(\frac{\mu_{1} \mu_{2} k_{1} t^{\alpha_{1}-1}}{1-\mu_{1} \mu_{2} k_{1} k_{2}} \int_{0}^{1} d A_{2}(t)+1\right) s(1-s)^{\alpha_{1}-1} \\
& \leq \frac{1}{\Gamma\left(\alpha_{1}-1\right)}\left(\frac{\mu_{1} \mu_{2} k_{1}}{1-\mu_{1} \mu_{2} k_{1} k_{2}} \int_{0}^{1} d A_{2}(t)+1\right) s(1-s)^{\alpha_{1}-1} \\
& \leq \rho s(1-s)^{\alpha_{1}-1},
\end{aligned}
$$




$$
\begin{aligned}
& H_{2}(t, s) \\
& =\frac{\mu_{2} t^{\alpha_{2}-1}}{1-\mu_{1} \mu_{2} k_{1} k_{2}} \int_{0}^{1} G_{1}(t, s) d A_{2}(t) \\
& \leq \frac{s(1-s)^{\alpha_{1}-1}}{\Gamma\left(\alpha_{1}-1\right)} \frac{\mu_{2} t^{\alpha_{2}-1}}{1-\mu_{1} \mu_{2} k_{1} k_{2}} \int_{0}^{1} d A_{2}(t) \\
& \leq\left(\frac{\mu_{2}}{\Gamma\left(\alpha_{1}-1\right)\left(1-\mu_{1} \mu_{2} k_{1} k_{2}\right)} \int_{0}^{1} d A_{2}(t)\right) s(1-s)^{\alpha_{1}-1} \\
& \leq \rho s(1-s)^{\alpha_{1}-1} .
\end{aligned}
$$

As for the proof of (26), we have

$$
K_{2}(t, s), H_{1}(t, s) \leq \rho s(1-s)^{\alpha_{2}-1}, \quad t, s \in[0,1] ;
$$

that is, (20) holds.

By $[20]$, for any $t, s \in[0,1]$, we have

$$
\begin{aligned}
& \frac{(1-t) t^{\alpha_{i}-1} s(1-s)^{\alpha_{i}-1}}{\Gamma\left(\alpha_{i}\right)} \\
& \quad \leq G_{i}(t, s) \leq \frac{t^{\alpha_{i}-1}(1-t)}{\Gamma\left(\alpha_{i}-1\right)}, \quad i=1,2 .
\end{aligned}
$$

So, by (11) and (28), we have

$$
\begin{aligned}
& K_{1}(t, s) \\
& =\frac{\mu_{1} \mu_{2} k_{1} t^{\alpha_{1}-1}}{1-\mu_{1} \mu_{2} k_{1} k_{2}} \int_{0}^{1} G_{1}(t, s) d A_{2}(t)+G_{1}(t, s) \\
& \leq \frac{\mu_{1} \mu_{2} k_{1} t^{\alpha_{1}-1}}{1-\mu_{1} \mu_{2} k_{1} k_{2}} \int_{0}^{1} \frac{t^{\alpha_{1}-1}(1-t)}{\Gamma\left(\alpha_{1}-1\right)} d A_{2}(t) \\
& +\frac{t^{\alpha_{1}-1}(1-t)}{\Gamma\left(\alpha_{1}-1\right)} \\
& \leq \frac{\mu_{1} \mu_{2} k_{1} t^{\alpha_{1}-1}}{1-\mu_{1} \mu_{2} k_{1} k_{2}} \int_{0}^{1} \frac{1}{\Gamma\left(\alpha_{1}-1\right)} d A_{2}(t)+\frac{t^{\alpha_{1}-1}}{\Gamma\left(\alpha_{1}-1\right)} \\
& \leq \frac{1}{\Gamma\left(\alpha_{1}-1\right)}\left(\frac{\mu_{1} \mu_{2} k_{1}}{1-\mu_{1} \mu_{2} k_{1} k_{2}} \int_{0}^{1} d A_{2}(t)+1\right) t^{\alpha_{1}-1} \\
& \leq \rho t^{\alpha_{1}-1}, \\
& H_{2}(t, s) \\
& =\frac{\mu_{2} t^{\alpha_{2}-1}}{1-\mu_{1} \mu_{2} k_{1} k_{2}} \int_{0}^{1} G_{1}(t, s) d A_{2}(t) \\
& \leq \frac{\mu_{2} t^{\alpha_{2}-1}}{1-\mu_{1} \mu_{2} k_{1} k_{2}} \int_{0}^{1} \frac{t^{\alpha_{1}-1}(1-t)}{\Gamma\left(\alpha_{1}-1\right)} d A_{2}(t) \\
& \leq \frac{\mu_{2} t^{\alpha_{2}-1}}{1-\mu_{1} \mu_{2} k_{1} k_{2}} \int_{0}^{1} \frac{1}{\Gamma\left(\alpha_{1}-1\right)} d A_{2}(t) \\
& \leq \rho t^{\alpha_{2}-1} \text {. }
\end{aligned}
$$

Proceeding as for the proof of (29), we have

$$
\begin{gathered}
K_{2}(t, s) \leq \rho t^{\alpha_{2}-1}, \\
H_{1}(t, s) \leq \rho t^{\alpha_{1}-1}, \\
t \in[0,1]
\end{gathered}
$$

thus (21) holds.

On the other hand, it follows from (11) and (25) that

$$
\begin{aligned}
& K_{1}(t, s) \\
& =\frac{\mu_{1} \mu_{2} k_{1} t^{\alpha_{1}-1}}{1-\mu_{1} \mu_{2} k_{1} k_{2}} \int_{0}^{1} G_{1}(t, s) d A_{2}(t)+G_{1}(t, s) \\
& \geq \frac{\mu_{1} \mu_{2} k_{1} t^{\alpha_{1}-1}}{1-\mu_{1} \mu_{2} k_{1} k_{2}} \int_{0}^{1} \frac{(1-t) t^{\alpha_{1}-1} s(1-s)^{\alpha_{1}-1}}{\Gamma\left(\alpha_{1}\right)} d A_{2}(t) \\
& \geq\left(\frac{\mu_{1} \mu_{2} k_{1}}{\Gamma\left(\alpha_{1}\right)\left(1-\mu_{1} \mu_{2} k_{1} k_{2}\right)} \int_{0}^{1}(1-t) t^{\alpha_{1}-1} d A_{2}(t)\right) \\
& \cdot t^{\alpha_{1}-1} s(1-s)^{\alpha_{1}-1} \\
& \geq \varrho t^{\alpha_{1}-1} s(1-s)^{\alpha_{1}-1}, \\
& H_{2}(t, s) \\
& =\frac{\mu_{2} t^{\alpha_{2}-1}}{1-\mu_{1} \mu_{2} k_{1} k_{2}} \int_{0}^{1} G_{1}(t, s) d A_{2}(t) \\
& \geq \frac{\mu_{2} t^{\alpha_{2}-1}}{1-\mu_{1} \mu_{2} k_{1} k_{2}} \int_{0}^{1} \frac{(1-t) t^{\alpha_{1}-1} s(1-s)^{\alpha_{1}-1}}{\Gamma\left(\alpha_{1}\right)} d A_{2}(t) \\
& \geq\left(\frac{\mu_{2}}{\Gamma\left(\alpha_{1}\right)\left(1-\mu_{1} \mu_{2} k_{1} k_{2}\right)} \int_{0}^{1}(1-t) t^{\alpha_{1}-1} d A_{2}(t)\right) \\
& \cdot t^{\alpha_{2}-1} s(1-s)^{\alpha_{1}-1} \\
& \geq \varrho t^{\alpha_{2}-1} s(1-s)^{\alpha_{1}-1},
\end{aligned}
$$

which implies that (22) holds. Similarly, we also have

$$
\begin{gathered}
K_{2}(t, s) \geq \varrho t^{\alpha_{2}-1} s(1-s)^{\alpha_{2}-1}, \\
H_{1}(t, s) \geq \varrho t^{\alpha_{1}-1} s(1-s)^{\alpha_{2}-1} \\
t \in[0,1] .
\end{gathered}
$$

This completes the proof of the lemma.

From Lemma 5, we have the following conclusion. 
Remark 6. For $t, \tau, s \in[0,1]$, we have

$$
\begin{aligned}
& K_{i}(t, s) \geq \omega t^{\alpha_{i}-1} K_{i}(\tau, s), \\
& H_{i}(t, s) \geq \omega t^{\alpha_{i}-1} H_{i}(\tau, s), i=1,2, \\
& K_{1}(t, s) \geq \omega t^{\alpha_{1}-1} H_{2}(\tau, s), \\
& H_{2}(t, s) \geq \omega t^{\alpha_{2}-1} K_{1}(\tau, s), \\
& K_{2}(t, s) \geq \omega t^{\alpha_{2}-1} H_{1}(\tau, s), \\
& H_{1}(t, s) \geq \omega t^{\alpha_{1}-1} K_{2}(\tau, s),
\end{aligned}
$$

where $\omega=\varrho / \rho, 0<\omega<1$.

Throughout this paper, we will work in the space $X=$ $C[0,1] \times C[0,1]$, which is a Banach space if it is endowed with the norm

$$
\begin{gathered}
\|(u, v)\|=\max \{\|u\|,\|v\|\}, \\
\|u\|=\max _{0 \leq t \leq 1}|u(t)|, \\
\|v\|=\max _{0 \leq t \leq 1}|v(t)| .
\end{gathered}
$$

Let

$$
\begin{array}{r}
K=\left\{(u, v) \in X: u(t) \geq \omega t^{\alpha_{1}-1}\|u, v\|,\right. \\
\left.v(t) \geq \omega t^{\alpha_{2}-1}\|u, v\|, t \in[0,1]\right\} ;
\end{array}
$$

then $K$ is a cone in $X$. For $0<r<R$, denote

$$
\begin{gathered}
K_{[r, R]}=\{(u, v) \in K: r \leq\|u, v\| \leq R\}, \\
K_{r}=\{(u, v) \in K:\|u, v\|<r\} .
\end{gathered}
$$

In what follows, we list some conditions to be used later:

$\left(\mathbf{H}_{1}\right) f:(0,1) \times[0,+\infty) \times(0,+\infty) \rightarrow[0,+\infty)$ is continuous, $f(t, x, y)$ is nondecreasing in $x$ and nonincreasing in $y$, and there exist $\lambda_{1}, \delta_{1} \in(0,1)$ such that

$$
\begin{aligned}
& c^{\lambda_{1}} f(t, x, y) \leq f(t, c x, y), \quad x, y>0, c \in(0,1), \\
& f(t, x, c y) \leq c^{-\delta_{1}} f(t, x, y), \quad x, y>0, c \in(0,1) ;
\end{aligned}
$$

$g:(0,1) \times(0,+\infty) \times[0,+\infty) \rightarrow[0,+\infty)$ is continuous, $g(t, x, y)$ is nonincreasing in $x$ and nondecreasing in $y$, and there exist $\lambda_{2}, \delta_{2} \in(0,1)$ such that

$$
\begin{aligned}
& c^{\lambda_{2}} g(t, x, y) \leq g(t, x, c y), \quad x, y>0, c \in(0,1), \\
& g(t, c x, y) \leq c^{-\delta_{2}} g(t, x, y), \quad x, y>0, c \in(0,1) ;
\end{aligned}
$$

$\left(\mathbf{H}_{2}\right)$

$$
\begin{aligned}
& \int_{0}^{1} s(1-s)^{\alpha_{1}-1} f\left(s, 1, s^{\alpha_{2}-1}\right) d s<+\infty, \\
& \int_{0}^{1} s(1-s)^{\alpha_{2}-1} g\left(s, s^{\alpha_{1}-1}, 1\right) d s<+\infty .
\end{aligned}
$$

Remark 7. By $\left(\mathbf{H}_{1}\right)$, we have

$$
\begin{aligned}
& f\left(s, s^{\alpha_{2}-1}, 1\right) \leq f\left(s, 1, s^{\alpha_{2}-1}\right), \\
& g\left(s, 1, s^{\alpha_{1}-1}\right) \leq g\left(s, s^{\alpha_{1}-1}, 1\right) .
\end{aligned}
$$

This together with $\left(\mathbf{H}_{2}\right)$ yields

$$
\begin{aligned}
& \int_{0}^{1} s(1-s)^{\alpha_{1}-1} f\left(s, s^{\alpha_{2}-1}, 1\right) d s \\
& \quad \leq \int_{0}^{1} s(1-s)^{\alpha_{1}-1} f\left(s, 1, s^{\alpha_{2}-1}\right) d s<+\infty \\
& \int_{0}^{1} s(1-s)^{\alpha_{2}-1} g\left(s, 1, s^{\alpha_{1}-1}\right) d s \\
& \quad \leq \int_{0}^{1} s(1-s)^{\alpha_{1}-1} g\left(s, s^{\alpha_{1}-1}, 1\right) d s<+\infty .
\end{aligned}
$$

From the above assumptions $\left(\mathbf{H}_{0}\right)-\left(\mathbf{H}_{2}\right)$, for any $(u, v) \epsilon$ $K \backslash\{(0,0)\}$, we define an integral operator $T: K \backslash\{(0,0)\} \rightarrow X$ by

$$
\begin{aligned}
& T(u, v)(t) \\
& \quad=\left(T_{1}(u, v)(t), T_{2}(u, v)(t)\right), \quad 0 \leq t \leq 1,
\end{aligned}
$$

where

$$
\begin{aligned}
& T_{1}(u, v)(t) \\
& =\int_{0}^{1} K_{1}(t, s) f(s, u(s), v(s)) d s \\
& \quad+\int_{0}^{1} H_{1}(t, s) g(s, u(s), v(s)) d s \\
& T_{2}(u, v)(t) \\
& =\int_{0}^{1} K_{2}(t, s) g(s, u(s), v(s)) d s \\
& \quad+\int_{0}^{1} H_{2}(t, s) f(s, u(s), v(s)) d s .
\end{aligned}
$$

Now we claim that $T$ is well defined for $(u, v) \in K \backslash\{(0,0)\}$. In fact, for any $(u, v) \in K \backslash\{(0,0)\}$, we have

$$
\begin{gathered}
\omega t^{\alpha_{1}-1}\|(u, v)\| \leq u(t) \leq\|(u, v)\|, \\
\omega t^{\alpha_{2}-1}\|(u, v)\| \leq v(t) \leq\|(u, v)\|, \\
t \in[0,1] .
\end{gathered}
$$


Let $c$ be a positive number such that $\|(u, v)\| / c<1, c>1$. From $\left(\mathbf{H}_{1}\right)$ and (44), we have

$$
\begin{aligned}
& f(t, u(t), v(t)) \\
& \quad \leq f\left(t, c, \omega t^{\alpha_{2}-1}\|(u, v)\|\right) \leq c^{\lambda_{1}} f\left(t, 1, \frac{\omega\|(u, v)\|}{c} t^{\alpha_{2}-1}\right) \\
& \quad \leq c^{\lambda_{1}+\delta_{1}}(\omega\|(u, v)\|)^{-\delta_{1}} f\left(t, 1, t^{\alpha_{2}-1}\right), \\
& g(t, u(t), v(t)) \\
& \quad \leq g\left(t, \omega t^{\alpha_{1}-1}\|(u, v)\|, c\right) \leq c^{\lambda_{2}} g\left(t, \frac{\omega\|(u, v)\|}{c} t^{\alpha_{1}-1}, 1\right) \\
& \quad \leq c^{\lambda_{2}+\delta_{2}}(\omega\|(u, v)\|)^{-\delta_{2}} g\left(t, t^{\alpha_{1}-1}, 1\right) .
\end{aligned}
$$

Hence, for any $t \in[0,1]$, by Lemma 5 and (45), we have

$$
\begin{aligned}
& T_{1}(u, v)(t) \\
& =\int_{0}^{1} K_{1}(t, s) f(s, u(s), v(s)) d s \\
& \quad+\int_{0}^{1} H_{1}(t, s) g(s, u(s), v(s)) d s \\
& \leq \rho\left(c^{\lambda_{1}+\delta_{1}}(\omega\|(u, v)\|)^{-\delta_{1}} \int_{0}^{1} s(1-s)^{\alpha_{1}-1} f\left(s, 1, s^{\alpha_{2}-1}\right) d s\right. \\
& \quad+c^{\lambda_{2}+\delta_{2}}(\omega\|(u, v)\|)^{-\delta_{2}} \\
& \left.\quad \cdot \int_{0}^{1} s(1-s)^{\alpha_{2}-1} g\left(s, s^{\alpha_{1}-1}, 1\right) d s\right) \\
& <+\infty .
\end{aligned}
$$

Similarly, for any $t \in[0,1]$, we have

$$
\begin{aligned}
& T_{2}(u, v)(t) \\
& \leq \rho\left(c^{\lambda_{1}+\delta_{1}}(\omega\|(u, v)\|)^{-\delta_{1}} \int_{0}^{1} s(1-s)^{\alpha_{1}-1} f\left(s, 1, s^{\alpha_{2}-1}\right) d s\right. \\
& \quad+c^{\lambda_{2}+\delta_{2}}(\omega\|(u, v)\|)^{-\delta_{2}} \\
& \left.\quad \cdot \int_{0}^{1} s(1-s)^{\alpha_{2}-1} g\left(s, s^{\alpha_{1}-1}, 1\right) d s\right) \\
& <+\infty
\end{aligned}
$$

Together with the continuity of $K_{i}(t, s)$ and $H_{i}(t, s)(i=$ $1,2)$, it is easy to see that $T_{i}(u, v) \in C[0,1]$, for $(u, v) \in K \backslash$ $\{(0,0)\}$. Therefore $T: K \backslash\{(0,0)\} \rightarrow X$ is well defined.

Obviously, $(u, v)$ is a positive solution of system (2) if and only if $(u, v)$ is a fixed point of $T$ in $K \backslash\{(0,0)\}$.

Lemma 8. Assume that $\left(\mathbf{H}_{0}\right)-\left(\mathbf{H}_{2}\right)$ hold. Then $T: K_{\left[r_{1}, r_{2}\right]} \rightarrow$ $K$ is a completely continuous operator.
Proof. First, we show $T\left(K_{\left[r_{1}, r_{2}\right]}\right) \subseteq K$.

For any $(u, v) \in K_{\left[r_{1}, r_{2}\right]}, 0 \leq t, \tau \leq 1$, by Remark 6, we have $T_{1}(u, v)(t)$

$$
\begin{aligned}
= & \int_{0}^{1} K_{1}(t, s) f(s, u(s), v(s)) d s \\
& +\int_{0}^{1} H_{1}(t, s) g(s, u(s), v(s)) d s \\
\geq & \int_{0}^{1} \omega t^{\alpha_{1}-1} K_{1}(\tau, s) f(s, u(s), v(s)) d s \\
& +\int_{0}^{1} \omega t^{\alpha_{1}-1} H_{1}(\tau, s) g(s, u(s), v(s)) d s
\end{aligned}
$$$$
\geq \omega t^{\alpha_{1}-1}\left(\int_{0}^{1} K_{1}(\tau, s) f(s, u(s), v(s)) d s\right.
$$

$$
\left.+\int_{0}^{1} H_{1}(\tau, s) g(s, u(s), v(s)) d s\right)
$$

$\geq \omega t^{\alpha_{1}-1} T_{1}(u, v)(\tau)$,

$T_{1}(u, v)(t)$

$$
\begin{aligned}
&= \int_{0}^{1} K_{1}(t, s) f(s, u(s), v(s)) d s \\
&+\int_{0}^{1} H_{1}(t, s) g(s, u(s), v(s)) d s \\
& \geq \int_{0}^{1} \omega t^{\alpha_{1}-1} H_{2}(\tau, s) f(s, u(s), v(s)) d s \\
&+\int_{0}^{1} \omega t^{\alpha_{1}-1} K_{2}(\tau, s) g(s, u(s), v(s)) d s \\
& \geq \omega t^{\alpha_{1}-1}\left(\int_{0}^{1} H_{2}(\tau, s) f(s, u(s), v(s)) d s\right. \\
&\left.\quad+\int_{0}^{1} K_{2}(\tau, s) g(s, u(s), v(s)) d s\right) \\
& \geq \omega t^{\alpha_{1}-1} T_{2}(u, v)(\tau) .
\end{aligned}
$$

Then, we have

$$
\begin{aligned}
& T_{1}(u, v)(t) \geq \omega t^{\alpha_{1}-1}\left\|T_{1}(u, v)\right\|, \\
& T_{1}(u, v)(t) \geq \omega t^{\alpha_{1}-1}\left\|T_{2}(u, v)\right\| ;
\end{aligned}
$$

that is,

$$
T_{1}(u, v)(t) \geq \omega t^{\alpha_{1}-1}\left\|\left(T_{1}(u, v), T_{2}(u, v)\right)\right\| .
$$

In the same way as (48), we can prove that

$$
T_{2}(u, v)(t) \geq \omega t^{\alpha_{2}-1}\left\|\left(T_{1}(u, v), T_{2}(u, v)\right)\right\| .
$$

Therefore, we have $T\left(K_{\left[r_{1}, r_{2}\right]}\right) \subseteq K$.

Next, we show $T: K_{\left[r_{1}, r_{2}\right]} \rightarrow K$ is continuous. 
Let $\left(u_{n}, v_{n}\right),(u, v) \in K_{\left[r_{1}, r_{2}\right]}$, such that $\left\|\left(u_{n}, v_{n}\right)-(u, v)\right\|$ $\rightarrow 0(n \rightarrow+\infty)$. Obviously, $r_{1} \leq\|(u, v)\|,\left\|\left(u_{n}, v_{n}\right)\right\| \leq r_{2}$ for all $n$; choose $c$ such that $\|(u, v)\| / c<1,\left\|\left(u_{n}, v_{n}\right)\right\| / c<1$, $c>1$. So, by Lemma $5,\left(\mathbf{H}_{1}\right)$, and (43), we have

$$
\begin{aligned}
& \left|T_{1}\left(u_{n}, v_{n}\right)(t)-T_{1}(u, v)(t)\right| \\
& =\mid\left(\int_{0}^{1} K_{1}(t, s) f\left(s, u_{n}(s), v_{n}(s)\right) d s\right. \\
& \left.+\int_{0}^{1} H_{1}(t, s) g\left(s, u_{n}(s), v_{n}(s)\right) d s\right) \\
& -\left(\int_{0}^{1} K_{1}(t, s) f(s, u(s), v(s)) d s\right. \\
& \left.+\int_{0}^{1} H_{1}(t, s) g(s, u(s), v(s)) d s\right) \\
& \leq 2 \rho \int_{0}^{1} s(1-s)^{\alpha_{1}-1} \\
& \cdot\left|f\left(s, u_{n}(s), v_{n}(s)\right)+f(s, u(s), v(s))\right| d s \\
& +2 \rho \int_{0}^{1} s(1-s)^{\alpha_{2}-1} \\
& \cdot\left|g\left(s, u_{n}(s), v_{n}(s)\right)+g(s, u(s), v(s))\right| d s \\
& \leq \rho\left(c^{\lambda_{1}+\delta_{1}}\left((\omega\|(u, v)\|)^{-\delta_{1}}+\left(\omega\left\|\left(u_{n}, v_{n}\right)\right\|\right)^{-\delta_{1}}\right)\right. \\
& \cdot \int_{0}^{1} s(1-s)^{\alpha_{1}-1} f\left(s, 1, s^{\alpha_{2}-1}\right) d s \\
& +c^{\lambda_{2}+\delta_{2}}\left((\omega\|(u, v)\|)^{-\delta_{2}}+\left(\omega\left\|\left(u_{n}, v_{n}\right)\right\|\right)^{-\delta_{2}}\right) \\
& \left.\cdot \int_{0}^{1} s(1-s)^{\alpha_{2}-1} g\left(s, s^{\alpha_{1}-1}, 1\right) d s\right) \\
& \leq \rho\left(2 c^{\lambda_{1}+\delta_{1}}\left(\omega r_{2}\right)^{-\delta_{1}} \int_{0}^{1} s(1-s)^{\alpha_{1}-1} f\left(s, 1, s^{\alpha_{2}-1}\right) d s\right. \\
& \left.+2 c^{\lambda_{2}+\delta_{2}}\left(\omega r_{2}\right)^{-\delta_{2}} \int_{0}^{1} s(1-s)^{\alpha_{2}-1} g\left(s, s^{\alpha_{1}-1}, 1\right) d s\right) \\
& <+\infty, \quad t \in[0,1] .
\end{aligned}
$$

By (52), for any $\varepsilon>0$, we can find a sufficiently large natural number $m>0$, for all $n$, such that

$$
\begin{aligned}
& \rho \int_{H(m)} s(1-s)^{\alpha_{1}-1} \\
& \cdot\left|f\left(s, u_{n}(s), v_{n}(s)\right)+f(s, u(s), v(s))\right| d s \\
&+\rho \int_{H(m)} s(1-s)^{\alpha_{2}-1} \\
& \cdot\left|g\left(s, u_{n}(s), v_{n}(s)\right)+g(s, u(s), v(s))\right| d s<\frac{\varepsilon}{2},
\end{aligned}
$$

where $H(m)=[0,1 / m] \cup[1-1 / m, 1]$. On the other hand, for each $(\bar{u}, \bar{v}) \in K_{\left[r_{1}, r_{2}\right]}$ and $t \in[1 / m, 1-(1 / m)]$, we have

$$
\begin{gathered}
\omega \omega r_{1} \leq \bar{u}(t), \\
\bar{v}(t) \leq r_{2},
\end{gathered}
$$

where $\Phi=\min \left\{t^{\alpha_{i}-1}: t \in[1 / m, 1-(1 / m)], i=1,2\right\}$. Since $f(t, x, y)$ and $g(t, x, y)$ are uniformly continuous in $[1 / m, 1-$ $(1 / m)] \times\left[\omega \bowtie r_{1}, r_{2}\right] \times\left[\omega \bowtie r_{1}, r_{2}\right]$, we have

$$
\begin{aligned}
& \lim _{n \rightarrow+\infty}\left|f\left(s, u_{n}(s), v_{n}(s)\right)-f(s, u(s), v(s))\right|=0, \\
& \lim _{n \rightarrow+\infty}\left|g\left(s, u_{n}(s), v_{n}(s)\right)-g(s, u(s), v(s))\right|=0
\end{aligned}
$$

hold uniformly on $s \in[1 / m, 1-(1 / m)]$. Then the Lebesgue dominated convergence theorem yields that

$$
\begin{array}{r}
\rho \int_{1 / m}^{1-(1 / m)} s(1-s)^{\alpha_{1}-1} \\
\cdot\left|f\left(s, u_{n}(s), v_{n}(s)\right)-f(s, u(s), v(s))\right| d s \longrightarrow 0, \\
\text { as } n \longrightarrow+\infty, \\
\rho \int_{1 / m}^{1-(1 / m)} s(1-s)^{\alpha_{2}-1} \\
\cdot\left|g\left(s, u_{n}(s), v_{n}(s)\right)-g(s, u(s), v(s))\right| d s \longrightarrow 0, \\
\text { as } n \longrightarrow+\infty .
\end{array}
$$

So, for the above $\varepsilon>0$, there exists a sufficiently large natural number $N_{0}$ such that when $n>N_{0}$, we have

$$
\begin{aligned}
\rho \int_{1 / m}^{1-(1 / m)} & s(1-s)^{\alpha_{1}-1} \\
\cdot & \mid f\left(s, u_{n}(s), v_{n}(s)\right) \\
& -f(s, u(s), v(s)) \mid d s<\frac{\varepsilon}{4}, \\
\rho \int_{1 / m}^{1-(1 / m)} & s(1-s)^{\alpha_{2}-1} \\
\cdot & \mid g\left(s, u_{n}(s), v_{n}(s)\right) \\
& -g(s, u(s), v(s)) \mid d s<\frac{\varepsilon}{4} .
\end{aligned}
$$


It follows from (53) and (57) that

$$
\begin{aligned}
& \left\|T_{1}\left(u_{n}, v_{n}\right)-T_{1}(u, v)\right\| \\
& \leq \int_{0}^{1} K_{1}(t, s) \mid f\left(s, u_{n}(s), v_{n}(s)\right) \\
& \quad-f(s, u(s), v(s)) \mid d s \\
& +\int_{0}^{1} H_{1}(t, s) \mid g\left(s, u_{n}(s), v_{n}(s)\right) \\
& \quad-g(s, u(s), v(s)) \mid d s \\
& \leq \rho \int_{1 / m}^{1-(1 / m)} s(1-s)^{\alpha_{1}-1} \mid f\left(s, u_{n}(s), v_{n}(s)\right) \\
& +\rho \int_{1 / m}^{1-(1 / m)} s(1-s)^{\alpha_{2}-1} \mid g\left(s, u_{n}(s), v_{n}(s)\right) \\
& +\rho \int_{H(m)} s(1-s)^{\alpha_{2}-1} \mid g\left(s, u_{n}(s), v_{n}(s)\right) \\
& +\rho \int_{H(m)} s(1-s)^{\alpha_{1}-1} \mid f\left(s, u_{n}(s), v_{n}(s)\right) \\
& +f(s, u(s), v(s)) \mid d s \\
& +g(s, u(s), v(s)) \mid d s \\
& +f s
\end{aligned}
$$

$<\varepsilon, \quad n>N_{0}$.

This implies that the operator $T_{1}: K_{\left[r_{1}, r_{2}\right]} \rightarrow C[0,1]$ is continuous. Similarly, we can prove $T_{2}: K_{\left[r_{1}, r_{2}\right]} \rightarrow C[0,1]$ is continuous. So $T: K_{\left[r_{1}, r_{2}\right]} \rightarrow K$ is continuous.

Finally, we show $T: K_{\left[r_{1}, r_{2}\right]} \rightarrow K$ is compact.

Let $D \subset K_{\left[r_{1}, r_{2}\right]}$ be any bounded set; then, for any $(u, v) \in$ $D$, we have $r_{1} \leq\|(u, v)\| \leq r_{2}$. Choose $c$, such that $\|(u, v)\| / c<$ $1, c>1$. By (45), for any $(u, v) \in D, t \in[0,1]$, we have

$$
\begin{aligned}
& T_{i}(u, v)(t) \\
& \leq \rho\left(\int_{0}^{1} s(1-s)^{\alpha_{1}-1} f(s, u(s), v(s)) d s\right. \\
& \left.\quad+\int_{0}^{1} s(1-s)^{\alpha_{2}-1} g(s, u(s), v(s)) d s\right) \\
& \leq \rho\left(c^{\lambda_{1}+\delta_{1}}\left(\omega r_{1}\right)^{-\delta_{1}} \int_{0}^{1} s(1-s)^{\alpha_{1}-1} f\left(s, 1, s^{\alpha_{2}-1}\right) d s\right. \\
& \left.\quad+c^{\lambda_{2}+\delta_{2}}\left(\omega r_{1}\right)^{-\delta_{2}} \int_{0}^{1} s(1-s)^{\alpha_{2}-1} g\left(s, s^{\alpha_{1}-1}, 1\right) d s\right) \\
& <+\infty, \quad i=1,2 .
\end{aligned}
$$

So $T(D)$ is bounded in $X$.
In what follows, we show that $T_{i}(D)$ is equicontinuous. In fact, by (59), for any $\varepsilon>0$, there exists a sufficiently large natural number $m_{0}$, for all $(u, v) \in D$, such that

$$
\begin{aligned}
& \rho\left(\int_{H\left(m_{0}\right)} s(1-s)^{\alpha_{1}-1} f(s, u(s), v(s)) d s\right. \\
& \left.\quad+\int_{H\left(m_{0}\right)} s(1-s)^{\alpha_{2}-1} g(s, u(s), v(s)) d s\right)<\frac{\varepsilon}{4}
\end{aligned}
$$

Let

$$
\begin{gathered}
M_{0}=\max \left\{f(t, x, y): \frac{1}{m_{0}} \leq t \leq 1-\frac{1}{m_{0}},\right. \\
\left.\omega \omega_{0} r_{1} \leq x \leq r_{2}, \omega \omega_{0} r_{1} \leq y \leq r_{2}\right\}, \\
M_{0}^{\prime}=\max \left\{g(t, x, y): \frac{1}{m_{0}} \leq t \leq 1-\frac{1}{m_{0}},\right. \\
\left.\omega \omega_{0} r_{1} \leq x \leq r_{2}, \omega \omega_{0} r_{1} \leq y \leq r_{2}\right\},
\end{gathered}
$$

where $\omega_{0}=\min \left\{t^{\alpha_{i}-1}: t \in\left[1 / m_{0}, 1-\left(1 / m_{0}\right)\right], i=1,2\right\}>0$.

By the uniform continuity of $K_{1}(t, s), H_{1}(t, s)$ on $[0,1] \times$ $[0,1]$, for the above $\varepsilon>0$, there exists $\delta_{0}>0$ such that, for any $t_{1}, t_{2} \in[0,1],\left|t_{1}-t_{2}\right|<\delta_{0}, s \in\left[1 / m_{0}, 1-\left(1 / m_{0}\right)\right]$, we have

$$
\begin{aligned}
& \left|K_{1}\left(t_{1}, s\right)-K_{1}\left(t_{2}, s\right)\right|<\frac{\varepsilon}{4}\left(M_{0}\left(1-\frac{2}{m_{0}}\right)\right)^{-1}, \\
& \left|H_{1}\left(t_{1}, s\right)-H_{1}\left(t_{2}, s\right)\right|<\frac{\varepsilon}{4}\left(M_{0}^{\prime}\left(1-\frac{2}{m_{0}}\right)\right)^{-1} .
\end{aligned}
$$

Thus, when $t_{1}, t_{2} \in[0,1],\left|t_{1}-t_{2}\right|<\delta_{0}, s \in\left[1 / l_{0},\left(l_{0}-1\right) / l_{0}\right]$, for any $(u, v) \in D$, we get

$$
\begin{aligned}
& \left|T_{1}(u, v)\left(t_{1}\right)-T_{1}(u, v)\left(t_{2}\right)\right| \\
& =\mid \int_{0}^{1} K_{1}\left(t_{1}, s\right) f(s, u(s), v(s)) d s \\
& \quad+\int_{0}^{1} H_{1}\left(t_{1}, s\right) g(s, u(s), v(s)) d s \\
& \quad-\left(\int_{0}^{1} K_{1}\left(t_{2}, s\right) f(s, u(s), v(s)) d s\right. \\
& \left.\quad+\int_{0}^{1} H_{1}\left(t_{2}, s\right) g(s, u(s), v(s)) d s\right) \mid
\end{aligned}
$$




$$
\begin{aligned}
& \leq \int_{1 / m_{0}}^{1-\left(1 / m_{0}\right)}\left|K_{1}\left(t_{1}, s\right)-K_{1}\left(t_{2}, s\right)\right| \\
& \cdot|f(s, u(s), v(s))| d s \\
& +\int_{1 / m_{0}}^{1-\left(1 / m_{0}\right)}\left|H_{1}\left(t_{1}, s\right)-H_{1}\left(t_{2}, s\right)\right| \\
& +\int_{H\left(m_{0}\right)}\left|K_{1}\left(t_{1}, s\right)-K_{1}\left(t_{2}, s\right)\right| \\
& \cdot f(s, u(s), v(s)) d s \\
& +\int_{H\left(m_{0}\right)}\left|H_{1}\left(t_{1}, s\right)-H_{1}\left(t_{2}, s\right)\right| \\
& \cdot g(s, u(s), v(s)) d s \\
& \leq \frac{\varepsilon}{2}+2 \rho \int_{H\left(m_{0}\right)} s(1-s)^{\alpha_{1}-1} \\
& +2 \rho \int_{H\left(m_{0}\right)} s(1-s)^{\alpha_{2}-1} \\
& \cdot g(s, u(s), v(s)) d s<\varepsilon
\end{aligned}
$$

This means that $T_{1}(D)$ is equicontinuous. By the ArzelaAscoli theorem, $T_{1}(D)$ is a relatively compact set. In the same way, we can show that $T_{2}(D)$ is a relatively compact set. So $T: K_{\left[r_{1}, r_{2}\right]} \rightarrow K$ is compact.

From the above discussion, together with the fact that $T$ : $K_{\left[r_{1}, r_{2}\right]} \rightarrow K$ is continuous, we get that $T: K_{\left[r_{1}, r_{2}\right]} \rightarrow K$ is completely continuous. This completes the proof of the lemma.

In order to obtain the existence of positive solutions of system (2), we will use the following cone compression and expansion fixed point theorem.

Lemma 9 (see [21]). Let $P$ be a positive cone in a Banach space $E, \Omega_{1}$ and $\Omega_{2}$ are bounded open sets in $E, \theta \in \Omega_{1}, \bar{\Omega}_{1} \subset \Omega_{2}$, and $A: P \cap \bar{\Omega}_{2} \backslash \Omega_{1} \rightarrow P$ is a completely continuous operator. If the following conditions are satisfied:

$$
\begin{gathered}
\|A x\| \leq\|x\|, \quad \forall x \in P \cap \partial \Omega_{1}, \\
\|A x\| \geq\|x\|, \quad \forall x \in P \cap \partial \Omega_{2},
\end{gathered}
$$

or

$$
\begin{gathered}
\|A x\| \geq\|x\|, \quad \forall x \in P \cap \partial \Omega_{1}, \\
\|A x\| \leq\|x\|, \quad \forall x \in P \cap \partial \Omega_{2},
\end{gathered}
$$

then $A$ has at least one fixed point in $P \cap\left(\bar{\Omega}_{2} \backslash \Omega_{1}\right)$.

\section{Main Results}

Theorem 10. Assume that $\left(\mathbf{H}_{0}\right)-\left(\mathbf{H}_{2}\right)$ hold; then system (2) has at least one positive solution $\left(u^{*}, v^{*}\right)$, and there exists a real number $0<l<1$ satisfying

$$
\begin{gathered}
l t^{\bar{\alpha}-1} \leq u^{*}(t) \leq l^{-1} t^{\alpha^{*}-1} \\
l t^{\bar{\alpha}-1} \leq v^{*}(t) \leq l^{-1} t^{\alpha^{*}-1} \\
t \in[0,1]
\end{gathered}
$$

where $\bar{\alpha}=\max \left\{\alpha_{1}, \alpha_{2}\right\}, \alpha^{*}=\min \left\{\alpha_{1}, \alpha_{2}\right\}$.

Proof. First, we show that system (2) has at least one positive solution.

Choose $r, R$, such that

$$
\begin{aligned}
& 0<r \\
& \leq \min \left\{\left(\left(\frac{1}{3}\right)^{\alpha_{1}-1} \varrho \omega^{\lambda_{1}}\right.\right. \\
& \left.\left.\cdot \int_{0}^{1} s(1-s)^{\alpha_{1}-1} f\left(s, s^{\alpha_{1}-1}, 1\right) d s\right)^{1 /\left(1-\lambda_{1}\right)}, \frac{1}{2}\right\}, \\
& R \geq \max \left\{\left(\rho \int_{0}^{1} s(1-s)^{\alpha_{1}-1} f\left(s, 1, s^{\alpha_{2}-1}\right) d s\right.\right. \\
& +\rho \int_{0}^{1} s(1-s)^{\alpha_{2}-1} \\
& \left.\left.\cdot g\left(s, s^{\alpha_{1}-1}, 1\right) d s\right)^{1 /\left(1-\max \left\{\lambda_{1}, \lambda_{2}\right\}\right)}, \frac{1}{\omega}, 2\right\} .
\end{aligned}
$$

For any $(u, v) \in \partial K_{r}$, we have

$$
\begin{gathered}
r \omega t^{\alpha_{1}-1} \leq u(t) \leq r \\
r \omega t^{\alpha_{2}-1} \leq v(t) \leq r \\
t \in[0,1] .
\end{gathered}
$$

By Lemma 5, Remark 7, and $\left(\mathbf{H}_{1}\right)$, for any $(u, v) \in \partial K_{r}$, we get

$$
\begin{aligned}
& T_{1}(u, v)(t) \\
& =\int_{0}^{1} K_{1}(t, s) f(s, u(s), v(s)) d s \\
& \quad+\int_{0}^{1} H_{1}(t, s) g(s, u(s), v(s)) d s \\
& \geq \int_{0}^{1} K_{1}(t, s) f(s, u(s), v(s)) d s \\
& \geq \varrho \int_{0}^{1} t^{\alpha_{1}-1} s(1-s)^{\alpha_{1}-1} f\left(s, r \omega s^{\alpha_{1}-1}, r\right) d s
\end{aligned}
$$




$$
\begin{aligned}
& \geq \varrho \int_{0}^{1} t^{\alpha_{1}-1} s(1-s)^{\alpha_{1}-1} f\left(s, r \omega s^{\alpha_{1}-1}, 1\right) d s \\
& \geq \varrho t^{\alpha_{1}-1} r^{\lambda_{1}} \omega^{\lambda_{1}} \int_{0}^{1} s(1-s)^{\alpha_{1}-1} f\left(s, s^{\alpha_{1}-1}, 1\right) d s \\
& \geq\left(\frac{1}{3}\right)^{\alpha_{1}-1} \varrho r^{\lambda_{1}} \omega^{\lambda_{1}} \int_{0}^{1} s(1-s)^{\alpha_{1}-1} f\left(s, s^{\alpha_{1}-1}, 1\right) d s \\
& \geq r=\|(u, v)\|, \quad t \in\left[\frac{1}{3}, \frac{2}{3}\right] .
\end{aligned}
$$

This guarantees

$$
\|T(u, v)\| \geq\|(u, v)\|, \quad(u, v) \in \partial K_{r} .
$$

On the other hand, for any $(u, v) \in \partial K_{R}$, we have

$$
\begin{gathered}
R \omega t^{\alpha_{1}-1} \leq u(t) \leq R, \\
R \omega t^{\alpha_{2}-1} \leq v(t) \leq R, \\
t \in[0,1] .
\end{gathered}
$$

By Lemma 5, $\left(\mathbf{H}_{1}\right)$, and $\left(\mathbf{H}_{2}\right)$, for any $(u, v) \in \partial K_{R}, t \in[0,1]$, we get

$$
\begin{aligned}
T_{1}(u, v)(t) & \\
= & \int_{0}^{1} K_{1}(t, s) f(s, u(s), v(s)) d s \\
& +\int_{0}^{1} H_{1}(t, s) g(s, u(s), v(s)) d s \\
\leq & \rho \int_{0}^{1} s(1-s)^{\alpha_{1}-1} f\left(s, R, R \omega s^{\alpha_{2}-1}\right) d s \\
& +\rho \int_{0}^{1} s(1-s)^{\alpha_{2}-1} g\left(s, R \omega s^{\alpha_{1}-1}, R\right) d s \\
\leq & \rho \int_{0}^{1} s(1-s)^{\alpha_{1}-1} f\left(s, R, s^{\alpha_{2}-1}\right) d s \\
& +\rho \int_{0}^{1} s(1-s)^{\alpha_{2}-1} g\left(s, s^{\alpha_{1}-1}, R\right) d s \\
\leq & \rho R^{\lambda_{1}} \int_{0}^{1} s(1-s)^{\alpha_{1}-1} f\left(s, 1, s^{\alpha_{2}-1}\right) d s \\
\leq & \rho R^{\max \left\{\lambda_{1}, \lambda_{2}\right\}}\left(\int_{0}^{1} s(1-s)^{\alpha_{1}-1} f\left(s, 1, s^{\alpha_{2}-1}\right) d s\right. \\
& +\rho R^{\lambda_{2}} \int_{0}^{1} s(1-s)^{\alpha_{2}-1} g\left(s, s^{\alpha_{1}-1}, 1\right) d s \\
& \\
& \\
&
\end{aligned}
$$

$\leq R=\|(u, v)\|$
In the same way as (72), we have

$$
T_{2}(u, v)(t) \leq R=\|(u, v)\|, \quad(u, v) \in \partial K_{R} .
$$

So,

$$
\|T(u, v)\| \leq\|(u, v)\|, \quad(u, v) \in \partial K_{R} .
$$

It follows from (70), (74), and Lemmas 8 and 9 that $T$ has a fixed point $\left(u^{*}, v^{*}\right)$ with $0<r \leq\left\|\left(u^{*}, v^{*}\right)\right\| \leq R$. It is easy to see that $\left(u^{*}, v^{*}\right)$ is a positive solution of system $(2)$.

Next, we show there exists a real number $0<l<1$ such that the positive solution $\left(u^{*}, v^{*}\right)$ in system (2) satisfies

$$
\begin{gathered}
l t^{\bar{\alpha}-1} \leq u^{*}(t) \leq l^{-1} t^{\alpha^{*}-1} \\
l t^{\bar{\alpha}-1} \leq v^{*}(t) \leq l^{-1} t^{\alpha^{*}-1} \\
t \in[0,1]
\end{gathered}
$$

where $\bar{\alpha}=\max \left\{\alpha_{1}, \alpha_{2}\right\}, \alpha^{*}=\min \left\{\alpha_{1}, \alpha_{2}\right\}$.

From Lemma 8 , we know $\left(u^{*}, v^{*}\right) \in K \backslash\{(0,0)\}$. So, we have

$$
\begin{gathered}
\omega t^{\alpha_{1}-1}\left\|\left(u^{*}, v^{*}\right)\right\| \leq u^{*}(t) \leq\left\|\left(u^{*}, v^{*}\right)\right\|, \\
\omega t^{\alpha_{2}-1}\left\|\left(u^{*}, v^{*}\right)\right\| \leq v^{*}(t) \leq\left\|\left(u^{*}, v^{*}\right)\right\|, \\
t \in[0,1] .
\end{gathered}
$$

Choose $c$, such that $\left\|\left(u^{*}, v^{*}\right)\right\| / c<1, c>1 / \omega$; by Lemma 5 and $\left(\mathbf{H}_{1}\right)$, for $t \in[0,1]$, we have

$$
\begin{aligned}
& u^{*}(t)=\int_{0}^{1} K_{1}(t, s) f\left(s, u^{*}(s), v^{*}(s)\right) d s \\
& +\int_{0}^{1} H_{1}(t, s) g\left(s, u^{*}(s), v^{*}(s)\right) d s \\
& \leq \int_{0}^{1} \rho t^{\alpha_{1}-1} f\left(s, c, \omega s^{\alpha_{2}-1}\left\|\left(u^{*}, v^{*}\right)\right\|\right) d s \\
& +\int_{0}^{1} \rho t^{\alpha_{1}-1} g\left(s, \omega t^{\alpha_{1}-1}\left\|\left(u^{*}, v^{*}\right)\right\|, c\right) d s \\
& \leq \rho t^{\alpha_{1}-1} \int_{0}^{1} f\left(s, c, \frac{\omega\left\|\left(u^{*}, v^{*}\right)\right\|}{c} s^{\alpha_{2}-1}\right) d s \\
& +\rho t^{\alpha_{1}-1} \int_{0}^{1} g\left(s, \frac{\omega\left\|\left(u^{*}, v^{*}\right)\right\|}{c} s^{\alpha_{1}-1}, c\right) d s \\
& \leq c^{\lambda_{1}+\delta_{1}}\left(\omega\left\|\left(u^{*}, v^{*}\right)\right\|\right)^{-\delta_{1}} \rho t^{\alpha_{1}-1} \int_{0}^{1} f\left(s, 1, s^{\alpha_{2}-1}\right) d s \\
& +c^{\lambda_{2}+\delta_{2}}\left(\omega\left\|\left(u^{*}, v^{*}\right)\right\|\right)^{-\delta_{2}} \rho t^{\alpha_{1}-1} \int_{0}^{1} g\left(s, s^{\alpha_{1}-1}, 1\right) d s \\
& \leq c^{\lambda_{1}+\delta_{1}}(\omega R)^{-\delta_{1}} \rho t^{\alpha_{1}-1} \int_{0}^{1} f\left(s, 1, s^{\alpha_{2}-1}\right) d s \\
& +c^{\lambda_{2}+\delta_{2}}(\omega R)^{-\delta_{2}} \rho t^{\alpha_{1}-1} \int_{0}^{1} g\left(s, s^{\alpha_{1}-1}, 1\right) d s \\
& \leq M t^{\alpha^{*}-1}
\end{aligned}
$$


where

$$
\begin{aligned}
M= & c^{\lambda_{1}+\delta_{1}}(\omega R)^{-\delta_{1}} \rho \int_{0}^{1} f\left(s, 1, s^{\alpha_{2}-1}\right) d s \\
& +c^{\lambda_{2}+\delta_{2}}(\omega R)^{-\delta_{2}} \rho \int_{0}^{1} g\left(s, s^{\alpha_{1}-1}, 1\right) d s .
\end{aligned}
$$

In the same way as (77), we also have $v(t) \leq M t^{\alpha^{*}-1}, t \in[0,1]$. Choose

$$
l=\min \left\{\omega r, \frac{1}{M}, \frac{1}{2}\right\}
$$

then we have

$$
\begin{gathered}
l t^{\bar{\alpha}-1} \leq u^{*}(t) \leq l^{-1} t^{\alpha^{*}-1} \\
l t^{\bar{\alpha}-1} \leq v^{*}(t) \leq l^{-1} t^{\alpha^{*}-1}, \\
t \in[0,1] .
\end{gathered}
$$

This completes the proof of Theorem 10 .

Theorem 11. Assume that $\left(\mathbf{H}_{0}\right)-\left(\mathbf{H}_{2}\right)$ hold. If $\lambda_{1}+\delta_{1}<1$ and $\lambda_{2}+\delta_{2}<1$, then system (2) has a unique positive solution for $\alpha_{1}=\alpha_{2}$.

Proof. Assume that system (2) has two different positive solutions $\left(u_{1}, v_{1}\right)$ and $\left(u_{2}, v_{2}\right)$. Denote $\alpha=\alpha_{1}=\alpha_{2}$; by Theorem 10, there exists $0<l_{1}<1,0<l_{2}<1$, such that

$$
\begin{gathered}
l_{1} t^{\alpha-1} \leq u_{1}(t) \leq \frac{1}{l_{1}} t^{\alpha-1}, \\
l_{1} t^{\alpha-1} \leq v_{1}(t) \leq \frac{1}{l_{1}} t^{\alpha-1}, \\
t \in[0,1] \\
l_{2} t^{\alpha-1} \leq u_{2}(t) \leq \frac{1}{l_{2}} t^{\alpha-1}, \\
l_{2} t^{\alpha-1} \leq v_{2}(t) \leq \frac{1}{l_{2}} t^{\alpha-1}, \\
t \in[0,1] .
\end{gathered}
$$

Thus, we have

$$
\begin{gathered}
l_{1} l_{2} u_{2}(t) \leq u_{1}(t) \leq \frac{1}{l_{1} l_{2}} u_{2}(t), \\
l_{1} l_{2} v_{2}(t) \leq v_{1}(t) \leq \frac{1}{l_{1} l_{2}} v_{2}(t), \\
t \in[0,1] .
\end{gathered}
$$

Obviously, $l_{1} l_{2} \neq 1$. Let

$$
\begin{aligned}
L=\sup \left\{l: l u_{2}(t) \leq u_{1}(t) \leq l^{-1} u_{2}(t),\right. \\
\left.l v_{2}(t) \leq v_{1}(t) \leq l^{-1} v_{2}(t), t \in[0,1]\right\} .
\end{aligned}
$$

It is easy to see that $0<l_{1} l_{2} \leq L<1$, and

$$
\begin{gathered}
L u_{2}(t) \leq u_{1}(t) \leq \frac{1}{L} u_{2}(t), \\
l v_{2}(t) \leq v_{1}(t) \leq \frac{1}{L} v_{2}(t), \\
t \in[0,1] .
\end{gathered}
$$

By $\left(\mathbf{H}_{1}\right)$, we have

$$
\begin{aligned}
& f\left(t, u_{1}(t), v_{1}(t)\right) \\
& \quad \geq f\left(t, L u_{2}(t), \frac{1}{L} v_{2}(t)\right) \geq L^{\lambda_{1}+\delta_{1}} f\left(t, u_{2}(t), v_{2}(t)\right) \\
& \quad \geq L^{\sigma} f\left(t, u_{2}(t), v_{2}(t)\right), \\
& g\left(t, u_{1}(t), v_{1}(t)\right) \\
& \quad \geq g\left(t, L u_{2}(t), \frac{1}{L} v_{2}(t)\right) \geq L^{\lambda_{2}+\delta_{2}} g\left(t, u_{2}(t), v_{2}(t)\right) \\
& \quad \geq L^{\sigma} g\left(t, u_{2}(t), v_{2}(t)\right),
\end{aligned}
$$

where $\sigma=\max \left\{\lambda_{1}+\delta_{1}, \lambda_{2}+\delta_{2}\right\}<1$. Consider the following:

$$
\begin{aligned}
& f\left(t, u_{2}(t), v_{2}(t)\right) \\
& \quad \geq f\left(t, L u_{1}(t), \frac{1}{L} v_{1}(t)\right) \geq L^{\lambda_{1}+\delta_{1}} f\left(t, u_{1}(t), v_{1}(t)\right) \\
& \quad \geq L^{\sigma} f\left(t, u_{1}(t), v_{1}(t)\right), \\
& g\left(t, u_{2}(t), v_{2}(t)\right) \\
& \quad \geq g\left(t, L u_{1}(t), \frac{1}{L} v_{1}(t)\right) \geq L^{\lambda_{2}+\delta_{2}} g\left(t, u_{1}(t), v_{1}(t)\right) \\
& \quad \geq L^{\sigma} g\left(t, u_{1}(t), v_{1}(t)\right) .
\end{aligned}
$$

From (85), for $t \in[0,1]$, we have

$$
\begin{aligned}
u_{1}(t) & \\
= & T_{1}\left(u_{1}, v_{1}\right)(t)=\int_{0}^{1} K_{1}(t, s) f\left(s, u_{1}(s), v_{1}(s)\right) d s \\
& +\int_{0}^{1} H_{1}(t, s) g\left(s, u_{1}(s), v_{1}(s)\right) d s \\
\geq & L^{\sigma} \int_{0}^{1} K_{1}(t, s) f\left(s, u_{2}(s), v_{2}(s)\right) d s \\
& +L^{\sigma} \int_{0}^{1} H_{1}(t, s) g\left(s, u_{2}(s), v_{2}(s)\right) d s \\
\geq & L^{\sigma} T_{1}\left(u_{2}, v_{2}\right)(t)=L^{\sigma} u_{2}(t),
\end{aligned}
$$


$u_{2}(t)$

$$
\begin{aligned}
= & T_{1}\left(u_{2}, v_{2}\right)(t)=\int_{0}^{1} K_{1}(t, s) f\left(s, u_{2}(s), v_{2}(s)\right) d s \\
& +\int_{0}^{1} H_{1}(t, s) g\left(s, u_{2}(s), v_{2}(s)\right) d s \\
\geq & L^{\sigma} \int_{0}^{1} K_{1}(t, s) f\left(s, u_{1}(s), v_{1}(s)\right) d s \\
& +L^{\sigma} \int_{0}^{1} H_{1}(t, s) g\left(s, u_{1}(s), v_{1}(s)\right) d s \\
\geq & L^{\sigma} T_{1}\left(u_{1}, v_{1}\right)(t)=L^{\sigma} u_{1}(t) .
\end{aligned}
$$

Similarly, from (86), we also have

$$
\begin{gathered}
v_{1}(t) \geq L^{\sigma} v_{2}(t), \\
v_{2}(t) \geq L^{\sigma} v_{1}(t), \\
t \in[0,1] .
\end{gathered}
$$

Therefore, we obtain

$$
\begin{gathered}
L^{\sigma} u_{2}(t) \leq u_{1}(t) \leq \frac{1}{L^{\sigma}} u_{2}(t), \\
L^{\sigma} v_{2}(t) \leq v_{1}(t) \leq \frac{1}{L^{\sigma}} v_{2}(t), \\
t \in[0,1] .
\end{gathered}
$$

Notice that $0<L<1,0<\sigma<1$; we have $L^{\sigma}>L$, so it is a contradiction with the maximality of $L$. Therefore, system (2) has a unique positive solution $\left(u^{*}, v^{*}\right)$. This completes the proof of Theorem 11.

Remark 12. By the proof of Theorems 10 and 11, we obtained the positive solutions of system (2) suppose $\left(\mathbf{H}_{0}\right)-\left(\mathbf{H}_{2}\right)$ hold; and the uniqueness of the solution to the system is established provided that system (2) satisfies the additional conditions $\left(\lambda_{1}+\delta_{1}<1\right.$ and $\left.\lambda_{2}+\delta_{2}<1\right)$ in theorem.

\section{Example}

Now we consider the existence and uniqueness of positive solutions for the fractional differential system (1). Obviously,

$$
\begin{gathered}
\alpha_{1}=\alpha_{2}=\frac{5}{2}, \\
\mu_{1}=\frac{1}{2}, \\
\mu_{2}=1, \\
A_{1}(t)=t, \\
A_{2}(t)=t^{1 / 2} .
\end{gathered}
$$

We also have

$$
\begin{gathered}
k_{1}=\int_{0}^{1} t^{\alpha_{2}-1} d A_{1}(t)=\int_{0}^{1} t^{3 / 2} d t=\frac{2}{5}>0, \\
k_{2}=\int_{0}^{1} t^{\alpha_{1}-1} d A_{2}(t)=\int_{0}^{1} t^{3 / 2} d t^{1 / 2} \\
=\frac{1}{2} \int_{0}^{1} t d t=\frac{1}{4}>0 \\
1-\mu_{1} \mu_{2} k_{1} k_{2}=\frac{19}{20}>0 .
\end{gathered}
$$

So, the condition $\left(\mathbf{H}_{0}\right)$ holds. For

$$
\begin{gathered}
f(t, x, y)=\frac{(\sin t+\cos t) \sqrt{x}}{\sqrt[3]{t(1-t) y}}, \\
g(t, x, y)=\frac{\sqrt[3]{y}}{e^{t} \sqrt{t(1-t) x}},
\end{gathered}
$$

it is easy to see that $f:(0,1) \times[0,+\infty) \times(0,+\infty) \rightarrow$ $(0,+\infty)$ is continuous, $f(t, x, y)$ is nondecreasing in $x$ and nonincreasing in $y, g:(0,1) \times(0,+\infty) \times[0,+\infty) \rightarrow$ $[0,+\infty)$ is continuous, and $g(t, x, y)$ is nonincreasing in $x$ and nondecreasing in $y$. Take

$$
\begin{aligned}
& \lambda_{1}=\frac{11}{20} \\
& \delta_{1}=\frac{2}{5} \\
& \lambda_{2}=\frac{3}{5} \\
& \delta_{2}=\frac{1}{5} .
\end{aligned}
$$

Then, we know that the condition $\left(\mathbf{H}_{1}\right)$ holds. As

$$
\begin{aligned}
& \int_{0}^{1} s(1-s)^{\alpha_{1}-1} f\left(s, 1, s^{\alpha_{2}-1}\right) d s \\
& \quad \leq 2 \int_{0}^{1} s^{1 / 2}(1-s) d s=\frac{8}{15}<+\infty, \\
& \int_{0}^{1} s(1-s)^{\alpha_{2}-1} g\left(s, s^{\alpha_{1}-1}, 1\right) d s \\
& \leq \int_{0}^{1} s^{-1 / 4}(1-s) d s=\frac{16}{21}<+\infty,
\end{aligned}
$$

the condition $\left(\mathbf{H}_{2}\right)$ also holds.

Therefore, by Theorem 10, we get that system (1) has at least one positive solution $\left(u^{*}, v^{*}\right) \in K_{\left[r_{1}, r_{2}\right]}$. For

$$
\begin{gathered}
\alpha_{1}=\alpha_{2}, \\
\lambda_{1}+\delta_{1}=\frac{11}{20}+\frac{2}{5}=\frac{19}{20}<1, \\
\lambda_{2}+\delta_{2}=\frac{3}{5}+\frac{1}{5}=\frac{4}{5}<1,
\end{gathered}
$$


by Theorem 11, we get that $\left(u^{*}, v^{*}\right)$ is the unique positive solution of system (1).

Remark 13. The example not only implies that $f(t, x, y)$ can be singular at $t=0,1, y=0$, and $g(t, x, y)$ can be singular at $t=0,1, x=0$, but also indicates that there is a large number of functions that satisfy the conditions of the theorems which we discuss in this paper. Also, the conditions in our theorems are easy to check.

\section{Conclusions}

In this paper, the $(n-1,1)$-type singular fractional differential system with coupled boundary conditions has been investigated. Based on the well-known Guo-Krasnoselskii fixed point theorem, the existence of solutions for the $(n-1,1)$-type fractional differential system with coupled integral boundary conditions is presented; also the uniqueness of positive solution is established when certain additional conditions are satisfied. The example given demonstrates the effectiveness and feasibility of our results.

\section{Conflict of Interests}

The authors declare that there is no conflict of interests regarding the publication of this paper.

\section{Acknowledgments}

The first, second, and third authors were supported financially by the National Natural Science Foundation of China (11371221), the Specialized Research Foundation for the Doctoral Program of Higher Education of China (20123705110001), and the Program for Scientific Research Innovation Team in Colleges and Universities of Shandong Province. The fourth author was supported financially by the Australia Research Council through an ARC Discovery Project Grant.

\section{References}

[1] S. G. Samko, A. A. Kilbas, and O. I. Marichev, Fractional Integrals and Derivatives: Theory and Applications, Gordon and Breach Science Publishers, Yverdon, Switzerland, 1993.

[2] I. Podlubny, Fractional Differential Equations, Academic Press, San Diego, Calif, USA, 1999.

[3] A. A. Kilbas, H. M. Srivastava, and J. J. Trujillo, Theory and Applications of Fractional Differential Equations, vol. 204 of North-Holland Mathematics Studies, Elsevier Science B.V., Amsterdam, The Netherlands, 2006.

[4] V. Lakshmikantham, S. Leela, and J. Vasundhara Devi, Theory of Fractional Dynamic Systems, Cambridge Academic Publishers, Cambridge, UK, 2009.

[5] K. Diethelm, The Analysis of Fractional Differential Equations, Springer, Berlin, Germany, 2010.

[6] J. J. Nieto and J. Pimentel, "Positive solutions of a fractional thermostat model," Boundary Value Problems, vol. 2013, article 5, 2013.
[7] B. Ahmad and J. J. Nieto, "Existence results for nonlinear boundary value problems of fractional integrodifferential equations with integral boundary conditions," Boundary Value Problems, vol. 2009, Article ID 708576, 11 pages, 2009.

[8] R. P. Agarwal, D. O’Regan, and S. Staněk, "Positive solutions for mixed problems of singular fractional differential equations," Mathematische Nachrichten, vol. 285, no. 1, pp. 27-41, 2012.

[9] X. Zhang, L. Liu, and Y. Wu, "The uniqueness of positive solution for a singular fractional differential system involving derivatives," Communications in Nonlinear Science and Numerical Simulation, vol. 18, no. 6, pp. 1400-1409, 2013.

[10] S. Liu, G. Wang, and L. Zhang, "Existence results for a coupled system of nonlinear neutral fractional differential equations," Applied Mathematics Letters, vol. 26, no. 12, pp. 1120-1124, 2013.

[11] R. Li, H. Zhang, and H. Tao, "Unique solution of a coupled fractional differential system involving integral boundary conditions from economic model," Abstract and Applied Analysis, vol. 2013, Article ID 615707, 6 pages, 2013.

[12] N. Xu and W. Liu, "Iterative solutions for a coupled system of fractional differential-integral equations with two-point boundary conditions," Applied Mathematics and Computation, vol. 244, pp. 903-911, 2014.

[13] J. Henderson and R. Luca, "Existence and multiplicity of positive solutions for a system of fractional boundary value problems," Boundary Value Problems, vol. 2014, article 60, 2014.

[14] L. Zhang, B. Ahmad, and G. Wang, "The existence of an extremal solution to a nonlinear system with the right-handed Riemann-Liouville fractional derivative," Applied Mathematics Letters, vol. 31, pp. 1-6, 2014.

[15] X. Zhang, C. Mao, Y. Wu, and H. Su, "Positive solutions of a singular nonlocal fractional order differential system via Schauder's fixed point theorem," Abstract and Applied Analysis, vol. 2014, Article ID 457965, 8 pages, 2014.

[16] W. Liu, X. Yan, and W. Qi, "Positive solutions for coupled nonlinear fractional differential equations," Journal of Applied Mathematics, vol. 2014, Article ID 790862, 7 pages, 2014.

[17] C. Bai and J. Fang, "The existence of a positive solution for a singular coupled system of nonlinear fractional differential equations," Applied Mathematics and Computation, vol. 150, no. 3, pp. 611-621, 2004.

[18] J. Wang, H. Xiang, and Z. Liu, "Positive solution to nonzero boundary values problem for a coupled system of nonlinear fractional differential equations," International Journal of Differential Equations, vol. 2010, Article ID 186928, 12 pages, 2010.

[19] K. S. Miller and B. Ross, An Introduction to the Fractional Calculus and Fractional Differential Equations, John Wiley \& Sons, New York, NY, USA, 1993.

[20] C. Yuan, "Multiple positive solutions for $(n-1,1)$-type semipositone conjugate boundary value problems of nonlinear fractional differential equations," Electronic Journal of Qualitative Theory of Differential Equations, vol. 2010, no. 36, pp. 1-12, 2010.

[21] D. J. Guo and V. Lakshmikantham, Nonlinear Problems in Abstract Cones, Academic Press, New York, NY, USA, 1988. 


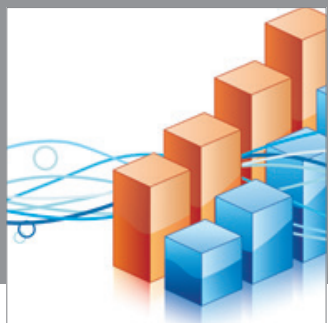

Advances in

Operations Research

mansans

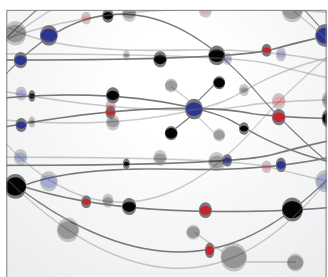

The Scientific World Journal
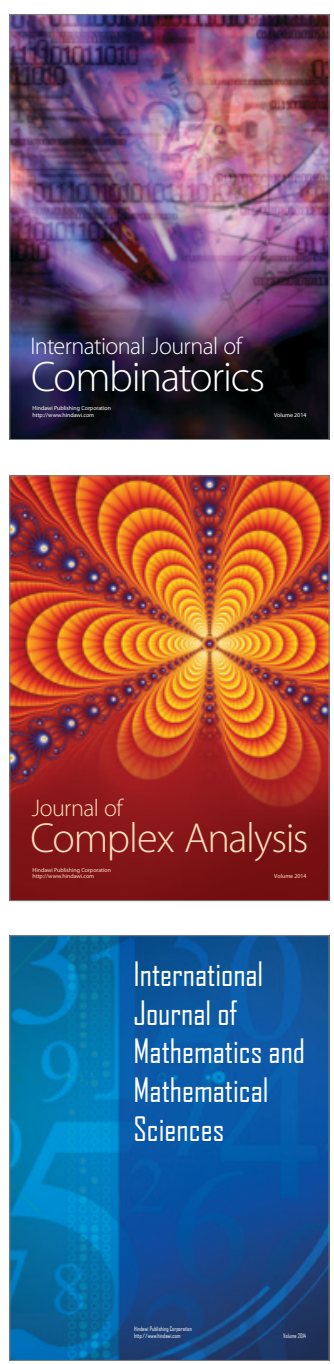
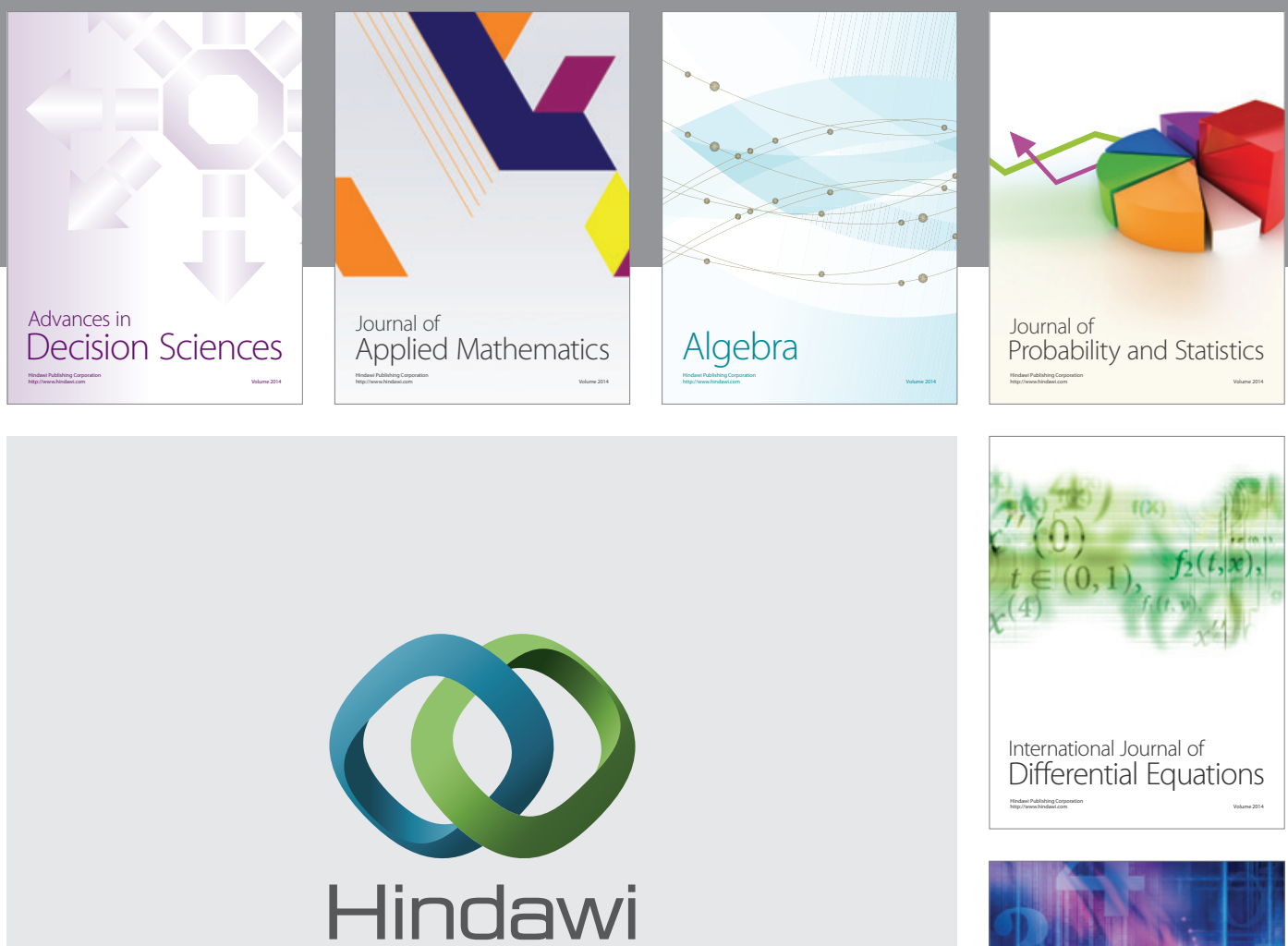

Submit your manuscripts at http://www.hindawi.com
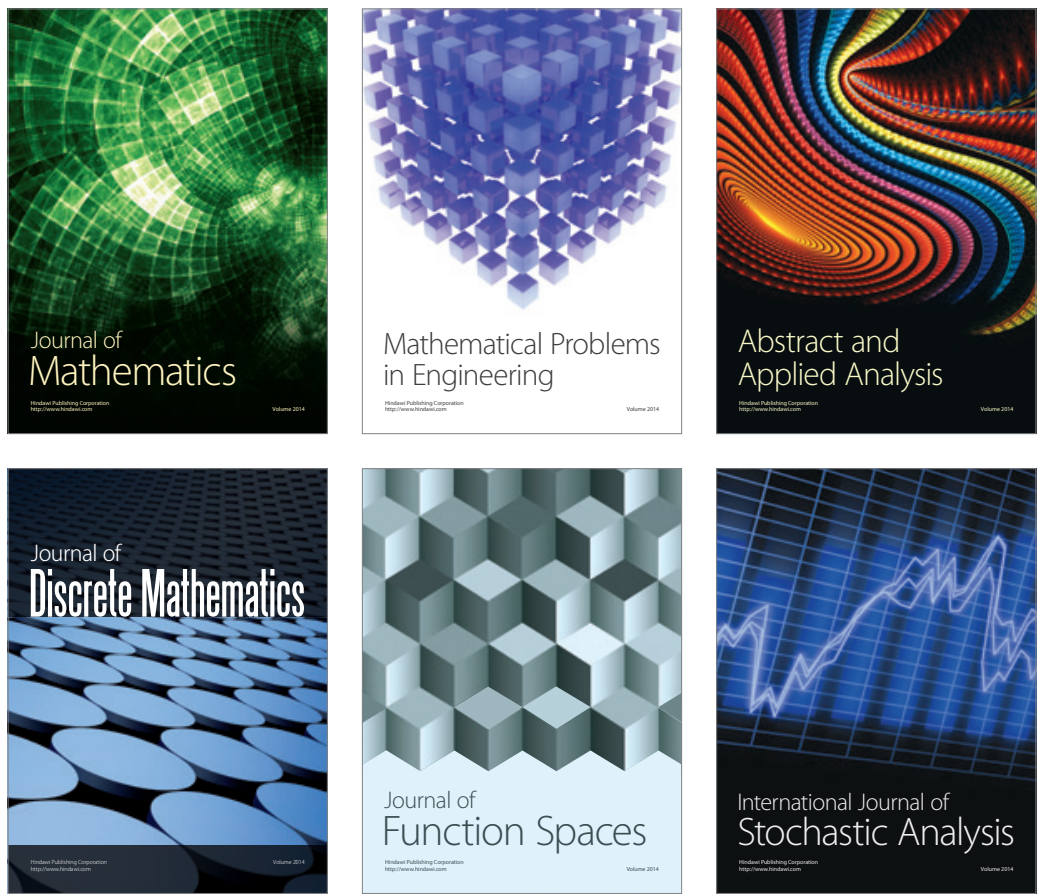

Journal of

Function Spaces

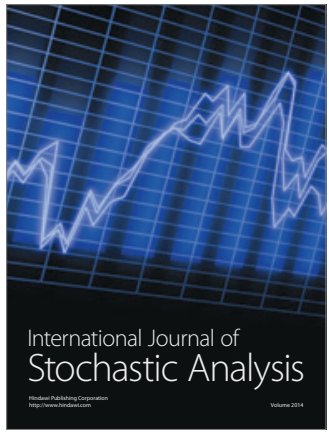

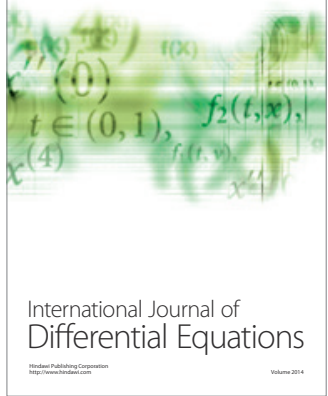
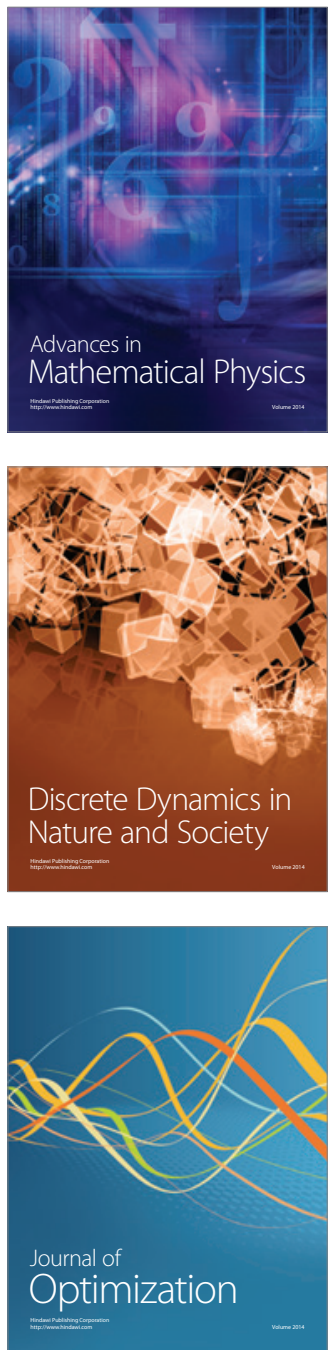